\title{
EMPREGO DO METARAMINOL NO BLOQUEIO DA HIPOTENSÃO PRODUZIDA PELO USO DA LEVOMEPROMAZINA EM CÃES
}

\author{
THE USE OF METARAMINOL BITARTARATE TO BLOCKADE THE HYPOTENSION PRODUCED BY THE \\ LEVOMEPROMAZINE HCL IN DOGS
}

Newton NUNES'; Luiz Gonzaga POMPERMAYER'; Josmari PIROLO; Sheila Canevese RAIIAL

\begin{abstract}
RESUMO
Avaliou-se a possibilidade do uso do bitartarato de metaraminol, visando o bloqueio da hipotensão produzida pelo cloridrato de levomepromazina em cães. Foram empregados 20 cães, machos e fêmeas, adultos, com pesos compreendidos entre $8 \mathrm{e} 12 \mathrm{~kg}$. Os animais foram divididos em dois grupos iguais (GI e GII). Aos cães do GI foi administrada, por via intravenosa, levomepromazina na dose de $2 \mathrm{mg} / \mathrm{kg}$, seguida, 15 minutos após, de $0,5 \mathrm{ml}$ de solução salina $0,9 \%$. A pressão arterial sistólica (PAS), freqüências cardíaca (FC) e respiratória (FR), temperatura retal $(\mathrm{T})$ e avaliação eletrocardiográfica na derivação DII foram observadas antes da aplicação da levomepromazina e após a mesma, em intervalos de 15 minutos durante 60 minutos. Aos cães do GII foi aplicada a mesma metodologia, substituindo-se, porém, a solução salina pelo metaraminol na dose de $0,1 \mathrm{mg} / \mathrm{kg}$, pela via intravenosa. Os valores obtidos foram submetidos à avaliação estatística pelo método de Análise de Perfil. Os resultados mostraram que o metaraminol produziu um aumento da PAS e diminuição da FC, além de promover menor queda da T e melhora dos valores de FR. Não foram observadas alterações na eletrocardiografia que pudessem ser atribuídas aos fármacos empregados. Concluiu-se que o metaraminol é seguro e indicado quando se emprega a levomepromazina, em cães.
\end{abstract}

UNITERMOS:Metaraminol; Levomepromazina; Anestesia; Cães

\section{INTRODUÇÃO E LITERATURA}

Existem ocasiões em que o profissional que se dedica à prática clínico-cirúrgica se depara com a necessidade da contenção farmacológica e potenciação do efeito de agentes anestésicos em pacientes portadores de hipotensão.

Dentre os tranqüilizantes de uso rotineiro destacam-se os fenotiazínicos. Os agentes deste grupo causam depressão do sistema nervoso central por sua ação sobre os centros nervosos inferiores, tálamo, hipotálamo e formação reticular, além de possuírem propriedades antieméticas e antiespasmódicas ${ }^{3}$.

Sobre a atividade cardiovascular os agentes do grupo determinam bloqueio alfa-adrenérgico dose-dependente, com diminuição da regulação vasomotora, levando à hipotensão $\mathrm{e}$ taquicardia, possuindo, entretanto, características antiarrítmicas ${ }^{11}$, propriedades estas acrescidas de efeito inotrópico negativo sobre o coração ${ }^{13}$.

A levomepromazina, em particular, é um agente considerado como da "série mista", possuindo, portanto, atividades antihistamínica e adrenolítica ${ }^{7}$, além de produzir discreta analgesia. Apesar de apresentar elevada margem de segurança, o uso do fármaco, a exemplo de outros do mesmo grupo, pode determinar vários níveis de hipotensão, elevando assim, o fator de risco da anestesia, principalmente no paciente em que a pressão arterial está aquém da normal.

O estudo da farmacodinâmica de agentes agonistas alfaadrenérgicos leva a crer que seu emprego, associado ao do fenotiazínico, pode inibir ou bloquear a vasodilatação produzida por este último. Neste sentido parece ser adequado o uso de metaraminol, uma droga simpatomimética de ação mista, isto é, que atua diretamente sobre receptores e indiretamente no ciclo da noradrenalina ${ }^{4,8,14}$. O metaraminol tem sido empregado no tratamento de estados de hipotensão ou no controle da taquicardia atrial paroxística, podendo-se aplicá-lo por via intravenosa, intramuscular em doses de 0,5 a $5,0 \mathrm{mg} / \mathrm{kg}^{4}$, embora LUMB; JONES ${ }^{6}$ (1984) recomendem doses entre 0,05 e $0,2 \mathrm{mg} / \mathrm{kg}$ pela via intravenosa ou 0,5 a 1,0 $\mathrm{mg} / \mathrm{kg}$ pela via intramuscular, no cão. A infusão contínua do fármaco também tem sido sugerida ${ }^{12}$.

Segundo COLLINS ${ }^{1}$ (1976), o período de latência do fármaco varia de 5 a 12 minutos e seu efeito perdura por 50 a 90

\footnotetext{
I - Professor Assistente - Faculdade de Ciências Agrárias e Veterinárias da UNESP-Jaboticabal, SP

2 - Professor Assistente - Universidade Federal de Viçosa-Viçosa, MG

3 - Professor Adjunto - Universidade Estadual de Londrina-Londrina, PR

4 - Professor Assistente - Faculdade de Medicina Veterinária e Zootecnia da UNESP-Botucatu, SP
} 
NUNES, N.; POMPERMAYER, L.G.; PIROLO, J.; RAHAL, S.C. Emprego do metaraminol no bloqueio da hipotensão produzida pelo uso da levomepromazina em cães. Braz. J. vet. Res. anim. Sci., São Paulo, v.32, n.2, p.120-4, 1995.

minutos. Já LUMB; JONES ${ }^{6}$ (1984) afirmam que o metaraminol inicia seus efeitos em 1 minuto e os mantém por 20 a 60 minutos. Embora a droga atue com mais eficiência nos vasos de resistência, os de capacitância também sofrem constrição, porém em menor grau, além de ser possível observar aumento do inotropismo do miocárdio ${ }^{10}$, tendo como resultante a elevação do débito cardíaco ${ }^{1.6}$.

Apesar do metaraminol ter ação marcante sobre os receptores alfa, tal efeito parece ser advindo do uso de altas doses, já que doses menores determinam estímulo beta-adrenoceptor ${ }^{5}$.

Devido à escassez de informações quanto ao emprego do metaraminol no controle da hipotensão induzida pela administração de fenotiazínicos, visou-se viabilizar seu uso como agente inibidor da hipotensão produzida, em cães, pelo emprego da levomepromazina.

\section{MATERIAL E MÉTODO}

Foram utilizados 20 cães adultos, sem raça definida, machos e fêmeas, com pesos compreendidos entre 8 e $12 \mathrm{~kg}$, considerados sadios, provenientes do canil da Faculdade de Medicina Veterinária e Zootecnia-FMVZ/UNESP, Campus de Botucatu, SP.

Após protocolados, os animais foram divididos, aleatoriamente, em dois grupos de 10 cães (GI e GII), aos quais foram administrados os seguintes tratamentos:

GI-Aplicação de cloridrato de levomepromazina*, na dose de $2 \mathrm{mg} / \mathrm{kg}$, pela via intravenosa e, após 15 minutos, aplicação, pela mesma via, de $0,5 \mathrm{ml}$ de solução salina $0,9 \%$ (placebo).

GII-Aplicação de levomepromazina na dose de $2 \mathrm{mg} / \mathrm{kg}$, pela via intravenosa e, após 15 minutos, administração, pela mesma via, de bitartarato de metaraminol **, na dose de 0,1 $\mathrm{mg} / \mathrm{kg}$ diluídos em solução salina $0,9 \%$ visando obter o volume total de $0,5 \mathrm{ml}$.

Os momentos estabelecidos para o delineamento experimental nos dois grupos foram os seguintes:

M1-15 minutos após a contenção física do animal, a fim de amenizar os efeitos do estresse inicial, e imediatamente antes da aplicação da levomepromazina.

M2-15 minutos após a administração da levomepromazina e imediatamente antes da aplicação do metaraminol (GII) ou placebo (GI).

\footnotetext{
* Neozine - Rhodia Farma Ltda

** Aramin - Laboratório Cristália Ltda

*** Estetoscópio Ultrasônico mod. EU 700 - Imbracrios Lida.

**** Eletrocardiógrafo FUNBEC mod. ECG5
}

M3- 30 minutos após M1.

M4- 45 minutos após M1.

M5- 60 minutos após M1.

\section{Variáveis fisiológicas mensuradas:}

a-Pressão arterial sistólica (PAS), obtida pelo método auscultatório, com o uso de esfigmomanômetro clínico, cuja parte inflável foi adaptada ao membro anterior, à altura do úmero e estetoscópio tipo Doppler***, cuja sonda foi posicionada sobre a artéria metacarpiana.

b-Eletrocardiografia na derivação DII, obtido com o emprego de eletrocardiógrafo $* * * *$.

c-Freqüência cardíaca (FC), obtida pela contagem do pulso da artéria femoral.

d-Freqüência respiratória (FR), pela observação direta dos movimentos respiratórios no nível das paredes torácica $\mathrm{e}$ abdominal.

e-Temperatura retal (T), pela introdução de termômetro clínico no reto.

Análise estatística foi efetuada por meio de Análise de Perfil $^{2,9}$ para interpretação dos possíveis efeitos que levaram à alteração nas médias de cada variável estudada nos diversos momentos, incluindo os testes das hipóteses de interação entre grupos e momentos, efeitos de grupos, efeitos de momentos, efeito de grupo em cada momento e efeito de momento dentro de cada grupo.

\section{RESULTADOS}

Decorridos 10 minutos da administração da levomepromazina, os animais perderam a refratariedade ao protocolo experimental. O efeito perdurou até a administração do metaraminol, quando imediatamente após a aplicação do fármaco alguns animais apresentaram comportamento mais agitado, caracterizado por movimentos dos membros e tentativa de levantar.

A análise estatística dos valores médios de PAS revelou perfis não similares ao longo do tratamento $(\mathrm{P}<0,001)$. Após administração do metaraminol (GII), observou-se diferença 
significativa entre os grupos em M3 (P<0,001), M4 (P<0,001) e M5 ( $\mathrm{P}<0,02)$. O estudo individual de cada grupo mostrou que após a administração da levomepromazina, houve queda significativa da PAS ( $<<0,001)$ em ambos os grupos, o que permaneceu inalterado no GI até o final de experimento. No GII, as médias observadas em M4 e M5 não diferem da observada em M1, porém, em M3 (15 minutos após o metaraminol), a média obtida foi significativamente maior ( $P$ $<0,001)$ que as verificadas nos demais momentos do grupo (Fig. 1).

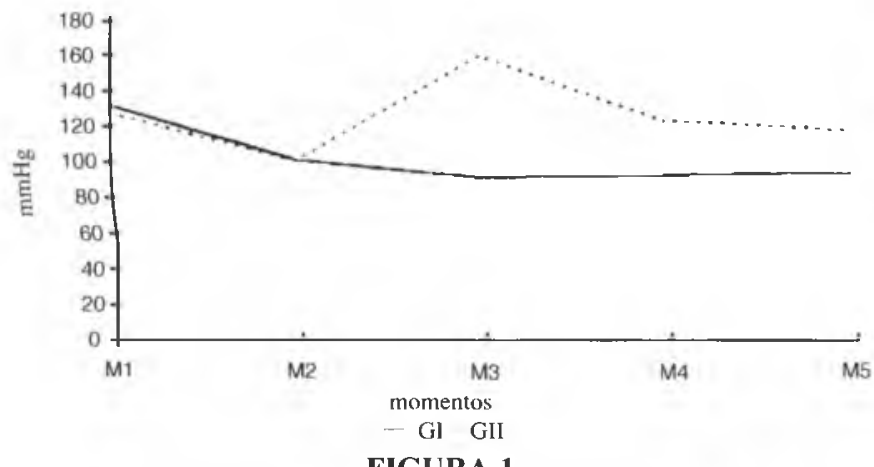

FIGURA 1

Variação dos valores médios da PAS $(\mathrm{mmHg})$, ao longo dos momentos, obtidos de cães tratados com levomepromazina e submetidos (GII) ou não (GI) ao metaraminol.

As médias de freqüência respiratória mostraram igualdade de perfis entre os grupos, com diferença significativa entre os momentos, no conjunto dos dois grupos $(\mathrm{p}<0,05)$. Após administração do metaraminol, observou-se diferença entre os grupos, com tendência à significância em M3 e M4 $(0,05<p<0,10)$, e significante em M5 $(p<0,05)$. O estudo individual de cada grupo mostrou no $\mathrm{Gl}$ igualdade entre M2 e M3, sendo ambos significativamente menores que Ml e maiores que M4, cuja média não diferiu de M5. No GII, as médias obtidas em M1 e M5 são iguais, mas foram significativamente superiores à obtida em M4 (Fig.2).

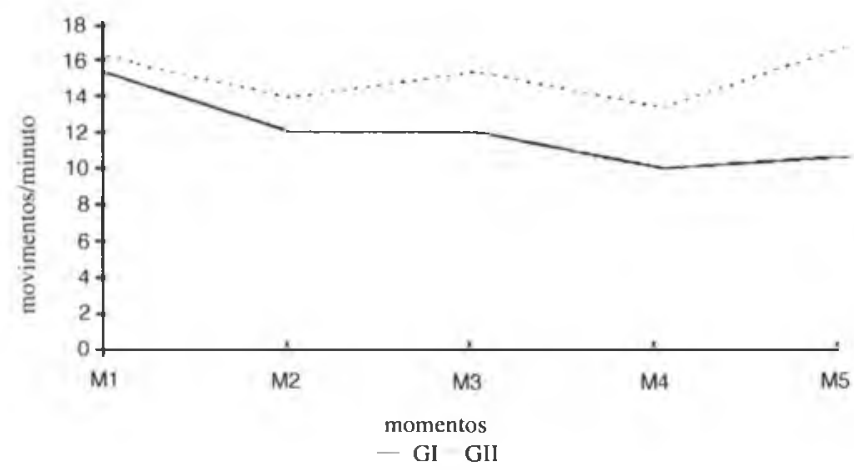

FIGURA 2

Variação dos valores médios de FR (movimentos/minuto), ao longo dos momentos, obtidas de cães tratados com levomepromazina e submetidos (GII) ou não (GI) ao metaraminol.
Os valores de freqüência cardíaca indicaram que os perfis dos dois grupos não são similares. O GII apresentou média significativamente maior ( $\mathrm{p}<0,01$ ) que GI em Ml, mas, nos demais momentos, os valores não apresentaram diferença significante entre os grupos. A análise individual de GII, revelou que após administração do metaraminol (M3) houve queda significativa $(\mathrm{p}<0,01)$ da $\mathrm{FC}$ em relação aos outros momentos do grupo (Fig.3).

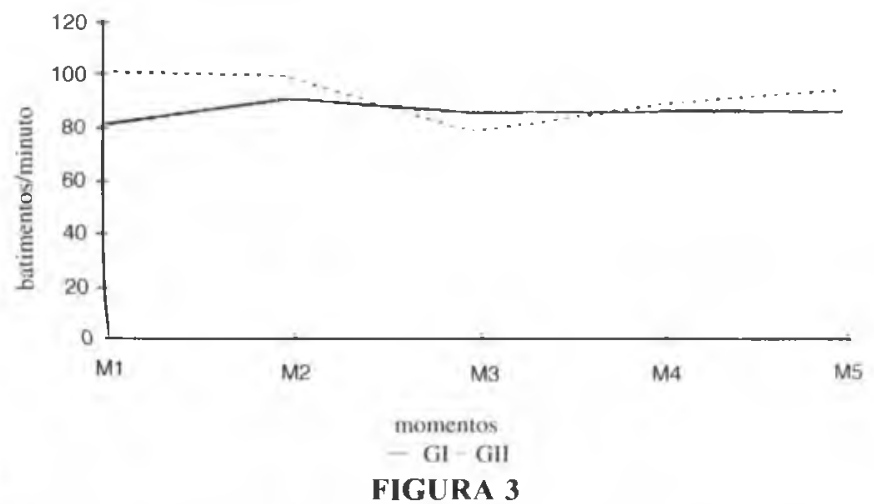

Variação dos valores médios da FC (batimentos/minuto), ao longo dos momentos, obtidas de cães tratados com levomepromazina e submetidos (GII) ou não (GI) ao metaraminol.

A temperatura retal ( $\mathrm{T}$ ) não mostrou perfis similares para os dois grupos. O GI apresentou média maior em M4 $(0,005<p<0,10)$, e em M5 ( $<<0,02)$. A análise individual de cada grupo revelou que no GI a temperatura diminuiu significativamente de momento para momento durante todo o experimento ( $\mathbf{p}<0,001$ ); já no GII, após a administração do metaraminol (de M3 a M5) as médias não variaram significativamente $(\mathrm{p}<0,001)$ entre os momentos (Fig. 4).

A avaliação eletrocardiográfica mostrou um animal com supradesnivelamento do intervalo S-T e outro com extrassístoles ventriculares ocasionais. Em ambos os casos a identificação da alteração foi feita em M1 do GII e as figuras eletrocardiográficas não se alteraram ao longo do experimento.

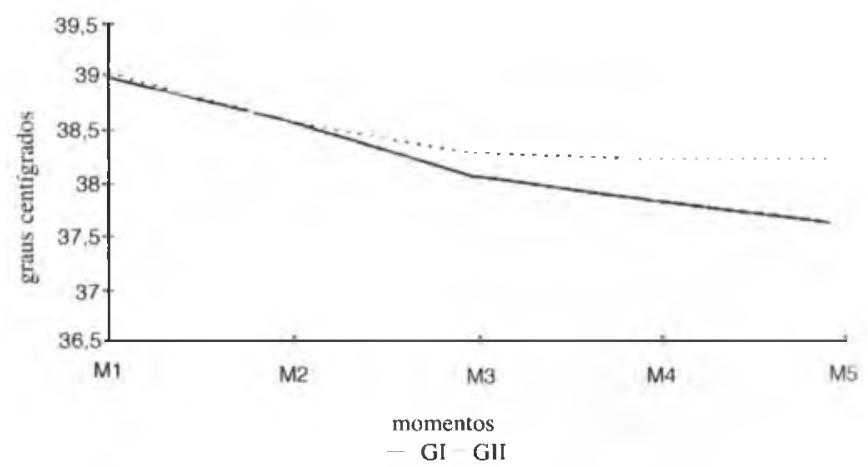

FIGURA 4

Variação dos valores médios da $\mathrm{T}\left({ }^{\circ} \mathrm{C}\right)$, ao longo dos momentos, obtidas de cães tratados com levomepromazina e submetidos (GIl) ou não (GI) ao metaraminol. 


\section{DISCUSSÃO}

O metaraminol foi capaz de antagonizar a hipotensão causada pela levomepromazina, produzindo, na verdade, hipertensão arterial, durante poucos minutos, fato este não comentado na literatura consultada. Como efeito mais importante, obtevese manutenção da PAS em níveis semelhantes aos basais até o final do experimento. Tal fato está de acordo com as assertivas de todos os autores, embora fosse possivel observar período de latência significativamente menor que o citado por COLLINS' (1976). Neste caso, o tempo necessário para que se pudesse observar efeito máximo foi o mesmo citado por LUMB; JONES ${ }^{6}$ (1984).

Embora a metodologia empregada não tenha objetivado estudar o efeito do agente simpatomimético em sítios específicos, sugere-se que a inibição da resposta hipotensora do fenotiazínico ocorra em conseqüência do aumento da liberação de noradrenalina provocado pelo metaraminol e/ou da competição desta droga com a levomepromazina pelos receptores alfa-adrenérgicos. De fato, não só a liberação de noradrenalina como a ação em receptores noradrenérgicos são efeitos atribuídos a drogas simpatomiméticas de ação mista, como o metaraminol ${ }^{8,14}$. Sobre a atividade cardíaca, a elevação da PAS, advinda da administração de metaraminol, determinou, por mecanismo reflexo, diminuição da $\mathrm{FC}$, como descrito por HOFFMAN; LEFKOWITZ4 (1987). Tal fato, associado ao efeito do agente simpatomimético também sobre receptores do tipo beta ${ }^{5}$ e a ação antiarrítmica da levomepromazina descrita por SHORT ${ }^{11}$ (1987), permite sugerir o uso associado dos dois fármacos. Neste particular, o inotropismo positivo produzido pelo metaraminol ${ }^{10}$ talvez possa sobrepujar o negativo advindo da aplicação da levomepromazina ${ }^{13}$. Até mesmo o efeito adrenolítico da fenotiazina, descrito por $\operatorname{MASSONE}^{7}$ (1986), apresenta balanço positivo com o efeito misto do metaraminol no que concerne à liberação acentuada de noradrenalina.

$\mathrm{O}$ estudo da freqüência respiratória permite verificar que mesmo sobre esta variável, o metaraminol age positivamente. Sendo assim, a queda da FR observada nos animais do GI e que pode ser explicada tanto pela queda do metabolismo basal advinda do uso da levomepromazina, como da posição de decúbito dorsal, foi inibida com o emprego do agente simpatomimético. O estudo da temperatura corpórea permite verificar que houve troca de calor com o meio ambiente e queda do metabolismo basal nos animais que receberam apenas a levomepromazina, ao contrário daqueles aos quais foi aplicado o metaraminol, quando estes efeitos não ocorreram. Parece claro que a vasoconstrição obtida nos cães do GII foi responsável pelo descrito.
$\mathrm{O}$ encontro de extra-sístoles ventriculares e supradesnivelamento do segmento $\mathrm{S}-\mathrm{T}$, observados em dois animais do GII, podem ser explicados como alterações individuais, não sendo possível incriminar qualquer dos fármacos, pois tais achados foram observados ainda em $\mathrm{Ml}$. Cabe, entretanto, salientar que nenhuma destas alterações foi acentuada com a metodologia adotada, o que, mais uma vez, demonstra a segurança clínica dos agentes estudados .

O comportamento agitado dos animais, observado após a administração de metaraminol, descrita neste trabalho, poderia ser atribuído à liberação de noradrenalina no sistema nervoso central $^{4}$; porém, há informações, na literatura, que afirmam ser esta droga desprovida de efeitos centrais ${ }^{8,14}$.

Por fim, pode-se sugerir que a aplicação do metaraminol em forma de infusão contínua, como citado por SILVA ${ }^{12}$ (1989), talvez constitua método mais adequado de administração, na medida em que as alterações iniciais bruscas da PAS e da FC poderão ser amenizadas e os valores obtidos tenderão a se manter constantes e dependentes da velocidade de administração.

\section{CONCLUSÃo}

Dos resultados obtidos deduz-se que a aplicação de metaraminol inibe a hipotensão induzida pela levomepromazina, sendo a associação dos fármacos segura e indicada para emprego em cães.

\section{AGRADECIMENTOS}

À disciplina de Anestesiologia Veterinária do Departamento de Cirurgia Veterinária e Reprodução Animal da FMVZ/ UNESP, Campus de Botucatu-SP, pela concessão das instalações e equipamentos utilizados.

Ao Prof. Dr. Paulo Roberto Curi, pela orientação e realização da análise estatística.

\section{OBSERVAÇÃO}

Não foi executado qualquer procedimento que pudesse ser considerado como desumano. Os cães não foram sacrificados ao final do experimento. 
NUNES, N.; POMPERMAYER, L.G.; PIROLO, J.; RAHAL, S.C. Emprego do metaraminol no bloqueio da hipotensão produzida pelo uso da levomepromazina em cães. Braz. J. vet. Res. anim. Sci., São Paulo, v.32, n.2, p.120-4, 1995.

\title{
SUMMARY
}

\begin{abstract}
The aim of this work was evaluate the possibility of the use of the metaraminol bitartarate to blockade the decrease of the blood pressure produced by levomepromazine HCL in dogs. To this 20 crossbreed adult dogs, males and females, with average weight from 8 to $12 \mathrm{~kg}$, were separated in two groups with $10 \mathrm{dogs}$ each (GI and GII). To the GI was administered, intravenously, $2 \mathrm{mg} / \mathrm{kg}$ of levomepromazine. Fifteen minutes after was administered, by the same way, $0.5 \mathrm{ml}$ of saline solution ( $0.9 \%)$. The systolic blood pressure (PA), heart rate $(\mathrm{FC})$, respiratory frequency $(\mathrm{FR})$, body temperature (T) and eletrocardiographic analysis (DII) was evaluated before and after the administration of the levomepromazine at 15 minutes of intervals, during 60 minutes. To the GIl was applied the same method but the saline solution was replaced by the metaraminol at $0.1 \mathrm{mg} / \mathrm{kg}$, administered by the intravenous way. The numeric data was evaluated by the profile analysis as statistic method. The results showed that the metaraminol increase the blood pressure and decrease the heart rate. The respiration and the body temperature were not changed with the use of metaraminol and the drugs administered did not produce eletrocardiographic alterations. The conclusion is that the use of metaraminol is indicatcd after the use of levomepromazine in dogs.
\end{abstract}

UNITERMS: Metaraminol; Levomepromazine; Anaesthesia; Dogs

\section{REFERÊNCIAS BIBLIOGRÁFICAS}

1-COLLINS, V.J. Principles of anesthesiology. 2.ed. Philadelphia, Lea \& Febiger, 1976.

2-CURI, P.R. Análise de medidas repetidas em experimentos biológicos. Revista Brasileira de Estatística, v. 41, n.161, p.137-50, 1980.

3-HALL, L.W.; CLARKE, K.W. Anestesia veterinária. 8.ed. São Paulo, Manole, 1987. p.56-9: Tranqüilizantes fenotiazínicos.

4-HOFFMAN, B.B.; LEFKOWITZ, R.J. Catecolaminas e drogas simpatomiméticas. In: GILMANN, A.G.; GOODMAN, L.S.; RALL, T.W.; MURAD, F. As bases farmacológicas da terapéutica. 7.ed. Rio de Janeiro, Guanabara, 1987. p. 123-44.

5-IWABUCHI, Y; AOKI, C.; MASUHARA, T. Effects of metaraminol on the secretion of fluid and glycoproteins from the rat submandibular gland. Japanase Journal Pharmacology, v.49, n. 4, p.491-500, 1989.

6-LUMB, W.V.; JONES, W.Veterinary anesthesia. 2.ed. Philadelphia, Lea \& Febiger, 1984.

7 - MASSONE, F.Anestesiologia veterinária. Farmacologia e técnicas. Rio de Janeiro, Guanabara, 1988.

08-MIZIARA, L.J. Adrenérgicos e antiadrenérgicos. In: SILVA, P. Farmacologia. 3.ed. Rio de Janeiro, Guanabara Koogan, 1989. p.1410.
9-MORRISON, D.F.Multivariate statistical methods. New York, McGraw-Hill, 1967.

10-SARNOFF, S.J.; CASE, R.B.; BERGLUND, E.; SARNOFF, L. Ventricular function. V. The cardiocirculatory effects of aramine; mechanism of action of "vasopressor" drugs in cardiogenic shock. Circulation, v.10, n.84, 1954 apud LUMB, W.V.; JONES, W.Veterinary anesthesia. 2.ed. Philadelphia, Lea \& Febiger, 1984. p.575.

11-SHORT, C.E. Principles \& practice in veterinary anesthesia. Baltimore, Willian \& Wilkins, 1987.

12-SILVA, P. Farmacologia. 3.ed. Rio de Janeiro, Guanabara Koogan, 1989.

13-SOMA, L.R. Textbook of veterinary anesthesia. Baltimore, Willian \& Wilkins, 1971.

14-WEINER, N. Noradrenalina, adrenalina e aminas simpatomiméticas. In: GILMAN, A.G.; GOODMAN, L.S.; RALL, T.W.; MURAD, F. As bases farmacológicas da terapêutica. 7. ed. Rio de Janeiro, Guanabara Koogan, 1985. p. 111 .

Recebido para publicação em 04/12/93 Aprovado para publicação em 12/09/94 